\title{
Perineal Travmayı Azaltmaya Yönelik Alternatif Yöntemler
}

\author{
Nurdan Kaya'®®, Gülbahtiyar Demirel²®
}

'Sivas Cumhuriyet Üniversitesi Suşehri Sağlık Yüksekokulu, Hemşirelik, Sivas, Türkiye

${ }^{2}$ Sivas Cumhuriyet Üniversitesi Cumhuriyet Üniversitesi Sağlık Bilimleri Fakültesi, Ebelik, Sivas, Türkiye

Nurdan Kaya, Msc, Öğr. Gör. Gülbahtiyar Demirel, Dr. Öğr. Üyesi

Illetişim:

Msc, Öğr. Gör. Nurdan Kaya

Sivas Cumhuriyet Üniversitesi Suşehri Sağlık Yüksekokulu, Hemşirelik, Sivas, Türkiye Tel: +905446756704

E-Posta:nrdn.kaya@windowslive.com

Gönderilme Tarihi : 10 Ağustos 2017

Revizyon Tarihi : 29 Eylül 2017

Kabul Tarihi : 26 Ekim 2017

\section{ÖZET}

Perineal travma nedenlerinden biri olan epizyotomi, dünya çapında 20. yüzyıl boyunca önemli derecede artış gösteren obstetrik cerrahi uygulamalardan biridir. Amerika Jinekoloji ve Obstetrik Birliği (2016) spontan vajinal doğumlarda epizyotominin rutin olarak uygulanmasını önermemekte ve epizyotomi uygulamasına fetüsün hızlıca doğurtulması gereken durumlarda, operatif vajinal doğumlarda ve omuz distozisi durumlarında başvurulması gerektiğini belirtmektedir. Yapılan güncel çalışmalarda dünyada epizyotomi sıklığı nulliparlar için \%50-\%95, multiparlar için \%6-20 arasında değişmektedir. Ülkemizde ise epizyotomi uygulanma sıklığına yönelik çalışmalar sınırlı sayıda olup, yapılan çalışmalarda epizyotomi uygulanma sıklı̆ı ı nullipar için $\% 90-\% 99$, multipar için $\% 50-\% 75$ arasındadır.

Günümüzde birçok güncel kanıt, sınırı epizyotomi uygulamasının rutin epizyotomi uygulamasına göre perineal travmalar açısından daha koruyucu olduğunu bildirmektedir. Bu doğrultuda literatürde perineal travma insidansını azaltmaya yönelik yapılan çeşitli uygulamalara rastlanmaktadır. Bu uygulamalar arasında perineal masaj, perineye sıcak uygulama, doğum pozisyonları, el manevraları ve ıkınma yöntemi yer almaktadır. Bu yöntemler perineal travmayı azaltan yöntemlerdir. Bu yöntemlerin uygulayıııları ise vajinal doğumda, çok önemli rol ve sorumluluklara sahip olan, ebeler ile doğum ve kadın hastalıkları hemşireleridir. Bu nedenle bu derleme çalışması, ilgili güncel literatür doğrultusunda epizyotomi, laserasyonlar gibi perineal travmaları azaltmaya yönelik yapılan alternatif yöntemlere dikkat çekerek konuya olan duyarlıı̆ı̆ı artırılması ve sağlık profesyonellerine yön gösterici olması amacıyla ele alınmıştır.

Anahtar sözcükler: Alternatif yöntem, doğum ve kadın hastalıkları hemşiresi, ebe, epizyotomi, perineal travma

\section{ALTERNATIVE METHODS FOR REDUCING PERINEAL TRAUMA}

\section{ABSTRACT}

Episiotomy, one of the causes of perineal trauma, is one of the world's leading obstetric surgical practices that have increased significantly during the 20th century. The American Gynecology and Obstetric Union (2016) does not recommend the routine application of episiotomies during spontaneous vaginal deliveries and recommends it in cases where a rapid delivery of the fetus is required, in operative vaginal deliveries and in shoulder dystocia cases. In current studies, the episiotomy frequency in the world varies between $50 \%$ and $95 \%$ for nulliparas and 6-20\% for multiparas. In our country, studies on the frequency of episiotomy are limited, but the application frequency of episiotomy is $\mathbf{9 0 - 9 9 \%}$ for nulliparas and $50 \%-75 \%$ for multiparas in those studies.

Today, much current evidence suggests that limited episiotomy is more protective than routine episiotomy in terms of perineal trauma. In this respect, there are various applications in the literature aimed at reducing the incidence of perineal trauma. These applications include perineal massage, warm application to the perineum, birth positions, hand maneuvers and push out. These methods are methods that reduce perineal trauma. The practitioners of these methods are midwives and obstetric and gynecological nurses who have a very important role and responsibility at/ during the vaginal birth. For this reason, this review study was carried out in order to increase the sensitivity to the subject and to guide health professionals by drawing attention to the alternative methods in order to reduce perineal traumas such as episiotomies and lacerations in accordance with the related current literature.

Keywords: Alternative method, obstetrics and gynecology nurse, midwife, episiotomy, perineal trauma 
$\mathbf{P}$ erineal travma (yaralanma), doğum esnasında perineal bölgede meydana gelen istemli (epizyotomi)/istemsiz (spontan perineal yırtıklar) hasarlardır. Perineal travma nedenlerinden birisi olan epizyotomi dünya çapında 20. yy boyunca önemli derecede artış gösteren obstetrik cerrahi uygulamalardan biridir. Epizyotomi çoğunlukla fetüsün doğumunu kolaylaştırmak, spontan ya da ciddi perineal yırtıkları önlemek amacıyla perine duvarına (cilt ve kas) insizyonla yapılan cerrahi uygulamadır (1). Yapılan güncel çalışmalarda dünyada epizyotomi sıklığı nulliparlar için \%50-\%95 (2-7), multiparlar için \%6-20 arasında değişmektedir $(3,4)$. Ülkemizde ise epizyotomi uygulanma sıklığına yönelik çalışmalar sınırlı sayıda olup, yapılan çalışmalarda epizyotomi uygulanma sıklığı nullipar için \%90-\%99, multipar için \%50-\%75 arasındadır (8-10). Amerika Jinekoloji ve Obstetrik Birliği (11), Kraliyet Koleji Jinekolog ve Obstetrisyenler Birliği (12) ve Kanada Obstetrik ve Jinekoloji Birliği (13) spontan vajinal doğumlarda epizyotominin rutin olarak uygulanmasını önermemektedir. Epizyotomi uygulamasına, fetüsün hızlıca doğurtulması gereken durumlarda, operatif vajinal doğumlarda ve omuz distozisi durumlarında başvurulması gerektiği belirtilmiştir $(11,14)$.

Günümüzde birçok güncel kanıt, sınırlı epizyotomi uygulamasının rutin epizyotomi uygulamasına göre perineal travmalar açısından daha koruyucu olduğunu bildirmektedir $(2-7,15,16)$. Bu doğrultuda literatürde perineal travma insidansını azaltmaya yönelik yapılan çeşitli uygulamalara rastlanmaktadır. Bu uygulamalar arasında perineal masaj, perineye sıcak uygulama, doğum pozisyonları, el manevraları ve ıkınma yöntemi yer almaktadır (10,17-29). Güncel olan bu yöntemlerin uygulanabilirliği ise vajinal doğumda çok önemli rol ve sorumluluklara sahip olan ebe ile doğum ve kadın hastalıkları hemşirelerine bağlıdır (10$12,15,17,19,20,23,25,26,28-31)$. Bu nedenle bu derleme çalışması, ilgili güncel literatür doğrultusunda epizyotomi, laserasyonlar gibi perineal travmaları azaltmaya yönelik yapılan alternatif yöntemlere dikkat çekerek konuya olan duyarlılığın artırılması ve sağlık profesyonellerine yön gösterici olması amacıyla ele alınmıştır.

\section{Epizyotominin sıklığı ve uygulandığı durumlar}

Epizyotomi uygulamasına; bebek başının kolay, hızlı ve tehlikesiz doğumunun sağlanması, fetal hipoksinin önlenmesi, doğumun aktif fazının kısaltılması, laserasyonların önlenmesi, anal sfinkterin korunması, doğumdan sonra perineye ilişkin sorunların önlenmesi (32) vb. nedenlerle primiparitede, gebelik 41 haftadan büyük olduğunda, doğum indüksiyonunda (augmented or induced), epidural analjezi ve oksitosin kullanımında, ikinci evrede litotomi pozisyonunda ve müdahaleli doğumda (instrumentation) sıklıkla başvurulmaktadır (33). Kamboçya'da yapılan bir çalışmada epizyotomi sıklığı \%94.5 olup, hekim ve ebelerin epizyotomi uygulama nedenleri arasında perineal yırtıklardan korkma, Asya'lı kadınların diğerlerinden daha kısa ve sert perineye sahip olduğu inanışı ve doğum odalarının aşırı kalabalık olmasından dolayı zaman kısıtlığı yer almaktadır (1). Buna karşılık Kwon ve Park (34) tarafından yapılan çalışmada, epizyotomi yapılan ve yapılmayan kadınlar arasında bir farklılığın olmadığı, epizyotomi uygulamasının perineal yaralanmalar için koruyucu olmadığı rapor edilmiştir. Yapılan diğer çalışmalarda epizyotomi sıklığı nulliparlar için; \%54 (3), \%55 (4), \%62 (6), \%62.1 (5) \%66 (7) ve \%92.3 (2), multiparlar için; \%6 (3), \%12 (4) oranlarında değişmektedir. Ülkemizde ise yapılan çalışmalarda epizyotomi uygulanma sıklığı nullipar ve mutipar doğumlar için; sırasıyla \%96,7 ve \%51,8 (10), \%95 ve \%60 (8), \%92 ve $\% 72$ (9) ve tüm doğumlar için; \%70.3 (10), \%69.7 (26) ve \%64 (8) olarak saptanmıştır.

\section{Sınırlı epizyotomi}

Sınırlı epizyotomi uygulamasının rutin epizyotomi uygulamasına göre daha yararlı olduğu bildirilmiştir (1116). Joshi ve Acharya (16) tarafindan primigravidalarda yapılan çalışmada rutin olarak epizyotomi yerine sınırlı epizyotomi uygulanmış olup bu oran \%22'dir. Çalışmaya alınan primigravidaların \%16.2'sinde intak perine (herhangi bir perine hasarı olmadığı), \%43.2'sinde birinci derece yırtık olduğu ve sadece bir gebede üçüncü derece yırtık oluştuğu (\%1.4) bulunmuştur. Doğum sonu dönemde de kadınlarda klinik bir komplikasyon gözlenmemiştir. Sekiz çalışmayı kapsayan 5541 gebeyi içeren Cochrane sistematik incelemesi sonuçlarına göre sınırlı epizyotomi uygulamasının rutin epizyotomi uygulamasına göre daha yararlı olduğu bildirilmiştir. Sınırlı epizyotomi uygulanan kadınların daha az şiddetli perineal travma, dikiş ve iyileşme komplikasyonlarının olduğu bulunmuştur. Ağrı durumu, üriner inkontinans, ağrılı cinsel ilişki, şiddetli vajinal ya da perineal travma yönünden ise fark bulunmamıştır (15).

\section{Epizyotomi uygulamasını azaltmaya yönelik alternatif uygulamalar}

Epizyotomi uygulaması sonrasında meydana gelen komplikasyonlar maternal sağlığı olumsuz yönde etkilemektedir. Ciddi vajinal/perineal travma, posterior perineal travma, kan kaybı, perineal ağrı, analjezi kullanımı, ağrıı cinsel ilişki, hematom, perine enfeksiyonu ve idrar kaçırma epizyotominin komplikasyonları arasında sayılmaktadır 
(15). Bu komplikasyonları önlemek ya da azaltmak amacıyla literatürde epizyotomi uygulamaların insidansını düşürmeye yönelik çeşitli uygulamalara rastlanmaktadır. Bu uygulamalar arasında perineal masaj $(10,17-20,23,25,26)$, perineye sıcak uygulama (17,35), doğum pozisyonları $(28,29,31,36-39)$, el manevraları $(17,21,22,24,40,41)$ ve ıkınma yöntemi $(27,30)$ yer almaktadır.

Perineal Masaj: Perine masajı vajenin yan duvarlarına parmaklar yardımıyla yapılan masaj olarak tanımlanmaktadır (10). Literatürde perineal masajın uygulanma zamanı ve tekniği ilgili çeşitli çalışmalar mevcuttur $(10,17-$ $19,20,23,25,26)$. Yapılan çalışmalarda perineal masaj; vajinanın yan duvarlarına iki parmak ile $(17,19)$, vajinanın 2-3 cm kadar içine iki başparmak ile yan duvarlara çengel şeklinde $(10,26)$, vajinanın 2-3 cm kadar içine posterior ve yan duvarlarına (18) ve vajinanın içine iki parmak ile $U$ şeklinde (25) yapılmıştır. Uygulanma zamanı olarak; prenatal dönemden başlanarak doğuma kadar yapılan çalışmalar $(10,18)$ ve sadece intrapartum dönemde yapılan çalışmalar yer almaktadır $(17,19,20,23,25,26)$. Yapılan çalışmalarda masajın uygulanması esnasında kayganlaştıııcı $(10,17,25)$, gliserin (26), E vitamini eklenmiş aynısefa (Calendula) yağı (18) ve lavanta yağı (19) kullanılmıştır.

Prenatal dönemden başlanarak yapılan perineal masajın etkinliği: Doğum öncesi dönemden başlanarak yapılan perineal masaj perine esnekliğini arttırmakta, perineal laserasyonları ve epizyotomi ihtiyacını azaltmaktadır $(10,23)$. Sayıner ve Demirci (10) tarafından yapılan çalışmada deney grubuna $(n=50) 34$. gebelik haftasından sonra başlanarak haftada 1-2 kez masaj yapılmış, kontrol grubuna $(n=50)$ herhangi bir müdahale yapılmamıştır. Deney ve kontrol grubunda sırasıyla; intak perine \%78 ve $\% 10$, epizyotomi $\% 10$ ve $\% 92$ ve laserasyon insidansı $\% 12$ ve $\% 10$ olarak bulunmuş ve çalışmanın sonucunda prenatal perine masajının perineal travmaları azalttığı belirtilmiştir. Mei-dan ve ark. (18) tarafından 203 nullipar (deney grubu $n=99$, kontrol grubu $n=104$ ) gebeyle yapılan çalışmada, deney grubundaki gebelere 34. gebelik hastasından başlayarak doğuma kadar her gün 10 dakika boyunca $\mathrm{E}$ vitamini eklenmiş aynısefa yağı ile masaj uygulanmıştır. Doğumun ikinci evresinde ebeler tarafından aynı yağ ile hem deney hem de kontrol grubuna masaj yapılmıştır. Çalışmada deney grubunun \%29.8'inde intak perine, \%20'sine epizyotomi uygulandığı, \%73.3'ünde birinci derece, \%26.7'sinde ikinci derece laserasyonların olduğu, üçüncü ve dördüncü derece laserasyonların olmadığı bulunmuştur. Kontrol grubunda ise \%40'ında intak perine, $\% 18.9^{\prime}$ unda epizyotomi uygulandığı, \%78.9'unda birinci derece, \%19.3'ünde ikinci derece, \%1.8'inde üçüncü ve dördüncü derece laserasyonların olduğu saptanmıştır. Çalışmada sonuç olarak prenatal perineal masajın perineal travmaları ne koruyucu nede zararlı bir etkisinin olmadığı sonucuna varılmıştır.

İntrapartum dönemde yapılan perineal masajın etkinliği: İntrapartum dönemde yapılan perineal masajın perine esnekliğini artırarak, perineal laserasyonları ve epizyotomi uygulamasını azalttığı yapılan çalışmalarla desteklenmektedir $(20,23)$. Perineal masaj ve teknikleri uygulanan 11.651 kadının dâhil olduğu sekiz randomize çalışmanın bulgularına göre, perineye sıcak uygulama, masaj uygulamanın 3. ve 4. derece yırtıkları ve epizyotomi ihtiyacını azalttığı bulunmuştur (20). Dört çalışmayı kapsayan 2497 kadını içeren Cochrane sistematik incelemesi sonuçlarına göre, masajın perine yaralanmalarını azalttığı belirtilmiştir (23). Albers ve ark. (17) tarafından 403 kadının (nullipar ve multipar) dâhil edildiği çalışmada doğumun ikinci evresinde ebeler tarafından kayganlaştırıcı ile perineal masaj yapılmış olup, kadınların \%23.3'ü intak perine, \%1.7'sine epizyotomi uygulandığı, birinci, ikinci, üçüncü ve dördüncü derece laserasyonların oranları sırasıyla $\% 22.6, \% 18.1, \% 1$ ve $\% 0.3$ olarak rapor edilmiştir. Attarha ve ark. (19) tarafından yapılan çalışmaya 176 nullipar kadın (Deney grubu $n=91$, kontrol grubu $n=85$ ) katılmışır. Uygulamaya servikal açıklık tamamlandığı zaman başlanmış, ebe tarafından lavanta yağı ile 5-10 dakika nazikçe masaj yapılmıştır. Kontrol grubuna sadece Ritgen manevrası uygulanmıştır. Çalışmada deney grubunda \%60.2'sinde intak perine, \%8'ine epizyotomi açıldığı ve \%31.8'inde laserasyon olduğu, kontrol grubunda ise \%2.4'ünde intak perine, \%80'nine epizyotomi açıldığı ve $\% 17.6$ 'sınde laserasyon olduğu belirlenmiştir. Lavanta yağı ile yapılan masajın doğumun ikinci evresinde epizyotomi ve laserasyonların derecesini düşürdüğü bulunmuştur. Zare ve ark. (25) tarafından yapılan çalışmaya 145 nullipar (deney grubu $n=45$, kontrol grubu $n=100$ ) kadın katılmıştır. Uygulamaya servikal açıklık tamamlandığında başlanmış ve ebe steril kayganlaştırıcı ile nazikçe 5-10 dakika masaj yapmış, ikinci evrede annenin ıkınma esnasında ve ıkınma aralarında masaja devam edilmiştir. Kontrol grubuna sadece Ritgen manevrası uygulanmıştır. Çalışmada deney grubunda \%22.2'sinde intak perine, \%44.4'ünde epizyotomi ve \%33.3'ünde laserasyon olduğu, kontrol grubunda ise \%20 .2'sinde intak perine, \%44.3'üne epizyotomi ve \%28.3'ünde laserasyon olduğu saptanmıştır. Steril kayganlaştırıcı ile yapılan masajın perineal travmaları azaltmaya yönelik bir etkisinin olmadığı sonucuna varılmıştır. Demirel ve Gölbaşı (26) tarafından 284 kadın (nullipar ve multipar) ile yapılan (deney grubu $n=142$, kontrol grubu $n=142$ ) çalışmada deney grubundaki kadınlara uygulayıcı tarafından, doğumun 1. evresinde her biri $10 \mathrm{dk}$. olmak üzere $4 \mathrm{kez}$, doğumun 
ikinci evresinde 1 kez gliserinle perine masajı yapılmıştır. Kontrol grubundaki kadınlar klinikteki rutin bakımı almıştır. Çalışmanın bulgularında; deney grubundaki kadınların \%31'ine, kontrol grubundaki kadınların ise \%69.7'sine epizyotomi açılmış, spontan perineal laserasyon oranları deney grubunda \%9.2, kontrol grubunda ise $\% 4.2$ olarak bulunmuş, doğum eyleminin 2. evre süre ortalaması deney grubunda 25.33 dk., kontrol grubunda $28.18 \mathrm{dk}$. olarak belirlenmiştir. Çalışmada doğum eyleminde uygulanan perine masajı laserasyon oluşma durumu açısından anlamlı bir farklılık yaratmamışken epizyotomi işlemini azalttığı, doğumun ikinci evresinin süresini kısalttığı sonucuna varılmıştır.

Perineye Sıcak Uygulama: Doğum boyunca yaygın olarak kullanılan sıcak kompres uygulamasının perineal travmaları azalttığına ve doğumun ikinci evresi boyunca konforu arttırdığına inanılmaktadır (35). Albers ve ark. (17) tarafından 404 kadının (nullipar ve multipar) dâhil edildiği çalışmada, ebeler tarafından doğumun ikinci evresinde perineye sıcak kompres uygulanan kadınlarda intak perine oranı \%23.3, epizyotomi uygulanma oranı ise $\% 0.3$ 'dür. Aynı çalışmada kadınların birinci, ikinci, üçüncü ve dördüncü derece laserasyonların oranları sırasıyla $\% 24.4, \% 17.3, \% 0.7$ ve $\% 0$ olarak rapor edilmiştir. Dahlen ve ark. (35) tarafından 717 nullipar (deney grubu $n=360$, kontrol grubu $n=357$ ) gebeyle yapılan çalışmada deney grubu ve kontrol grubu arasında epizyotomi uygulanma sıklığı açısından istatistiksel açıdan anlamlı bir fark olmadı, üçüncü ve dördüncü derece laserasyonları önemli derecede azalttığı belirtilmiştir.

Doğum Pozisyonları: Yüzyıllardır doğumda dik pozisyonlar ile yatar pozisyonların kadın için avantajlarının olup olmadığı ile ilgili tartışmalar söz konusudur $(36,38)$. Doğumda litotomi pozisyonu ile epizyotomi uygulaması yaygın olarak kullanılmaktadır (31). Buna karşın doğumun ikinci evresinde dik pozisyonlarda olan kadınlarda epizyotomi oranlarında anlamlı derecede azalma olduğu bildirilmiştir (29,36-38). Doğumda lateral pozisyon ile litotomi pozisyonunu karşılaştırıldığı bir çalışmada; lateral pozisyonun perineal tarvmalar açısından koruyucu olduğu, epizyotomiye daha az ihtiyaç duyulduğu belirtilmiştir (39). Başka bir çalışmada ise dik pozisyonlardan diz ve el diz pozisyonunun perineal travmaları önlemek açısından en koruyucu pozisyonların olduğu rapor edilmiştir (28).

El Manevraları: Doğumun ikinci evresinde fetüs başının doğurtulması esnasında perineyi korumaya yönelik el manevraları uygulanmaktadır. Ritgen manevrası olarak da bilinen "hands on" tekniğinde bir el fetal baş üzerindeyken diğer el ile perine desteklenir. "Hands off" tekniğinde ise fetal baş doğarken el ile müdahale edilmez, bu teknik fetal başın perineye el müdahalesi olmadan doğması esasına dayanmaktadır $(41,42)$. Albers ve ark. (17) tarafından 404 kadının (nullipar ve multipar) dâhil edildiği çalışmada doğumun ikinci evresinde ebeler tarafından perineyi korumaya yönelik "hands off" tekniği uygulanmıştır. Kadınların $\% 22.3$ 'ünde intak perine, $\% 0.5^{\prime}$ ine epizyotomi uygulandığı, birinci, ikinci, üçüncü ve dördüncü derece laserasyonların oranları sırasıyla $\% 23, \% 18.3, \% 0.5$ ve $\% 1$ olarak rapor edilmiştir. Nullipar kadınlar ile yapılan bir çalışmada "hands on" ve "hands off" tekniğinin epizyotomi ve laserasyon insidansı üzerine etkisinin olmadığı bulunmuştur (40). Nullipar kadınlarda yapılan çalışmalarda "hands off" tekniğinin "hands on" tekniğine kıyasla epizyotomi ve laserasyonların insidansını anlamlı olarak azalttığı belirtilmiştir $(21,24)$. El manevraları ve perineal masajın perine travmaları üzerine etkisini araştıran bir çalışmada "hands off" tekniğinin perineal travmaları önlemede daha etkili olduğu saptanmıştır (22).

Ikınma Yöntemi: Doğumun ikinci evresinde iki ıkınma tekniği vardır. Bunlar; açık glotis-nefes vererek ıkınma (spontan ıkınma), kapalı glotis-nefes tutarak ıkınmadır (valsalva tipi). Hangisinin anne ve bebek sağlığı açısından yararlı olduğu konusunda fikir birliği yoktur (27). Özellikle nullipar kadınlar için spontan ıkınma tekniklerinin kullanılmasının epizyotomi oranlarını azaltacağı belirtilmiştir (4). Yapılan sistematik bir incelemede de valsalva tipi ıkınmanın rutin olarak kullanılmaması gerektiği, spontan ıkınmanın daha iyi bir klinik uygulama olduğu kabul edilmiştir (30).

\section{Epizyotomiyi önlemeye yönelik yapılacak alternatif uygulamalarda ebe/doğum ve kadın hastalıkları hemşiresinin sorumlulukları}

Spontan vajinal doğumda ebe, doğum ve kadın hastalıkları hemşiresi epizyotomiyi önlemeye yönelik alternatif uygulamaları yaparak rutin epizyotomi yerine sınırlı epizyotomi uygulamasına destek vermelidir (11-13,15). Ebe, doğum ve kadın hastalıkları hemşiresi gerek prenatal dönemden başlayarak gerek intrapartum dönemde perinenin esnekliğini artıracak, laserasyon ve epizyotomi oranlarını azaltacak alternatif yöntemleri (perineal masaj, perineye sıcak uygulama, doğumda dik pozisyonların kullanımı, spontan ıkınma, hand off tekniği ile ıkınma vb.) gebeye/partnerine öğretmeli veya kendi uygulamalı, tercih etmesi için cesaretlendirmeli, anneyi desteklemelidir (17,27-31,37-39). Bunun yanında fetal ve maternal riskli bir durum görüldüğü takdirde epizyotomi yapmaktan da kaçınılmamalıdır. 


\section{Kaynaklar}

1. Schantz C, Sim KL, Ly EM, Barennes H, Sudaroth S, Goyet S. Reasons for Routine Episiotomy: A Mixed-Methods Study in a Large Maternity Hospital in Phnom Penh, Cambodia. Reprod Health Matters 2015; 23: 68-77. [CrossRef]

2. Althabe F, Belizan JM, Bergel E. Episiotomy Rates in Primiparous Women in Latin America: Hospital Based Descriptive Study. BMJ 2002; 324: 945-6.

3. Van den Bergh JE, Sueters M, Segaar M, Van Roosmalen J. Determinants of Episiotomy in Rural Zimbabwe. Acta Obstet Gynecol Scand 2003; 82: 966-8.

4. Raisanen S, Vehvilainen- Julkunen K, Heinonen S. Need for and Consequences of Episiotomy in Vaginal Birth: A Critical Approach. Midwifery 2010; 26: 348-56. [CrossRef]

5. Izuka EO, Dim CC, Chigbu CO, Obiora-Izuka CE. Prevalence and Predictors of Episiotomy Among Women at First Birth in Enugu, South-East Nigeria. Ann Med Health Sci Res 2014; 4: 928-32. [CrossRef]

6. Hernández Pérez J, Azón López E, Mir Ramos E, Peinado Berzosa R, Val Lechuz B, Mérida Donoso A. Factors Affecting the Performance of a Selective Episiotomy in Nulliparous Women. Julio 2014; 35: 412-24.

7. Al-Ghammari K, Al-Riyami Z, Al-Moqbali M, Al-Marjabi F, Al-Mahrouqi $\mathrm{B}, \mathrm{Al}-\mathrm{Khatri} \mathrm{A}$ et al. Predictors of Routine Episiotomy in Primigravida Women in Oman. Appl Nurs Res 2016; 29: 131-5. [CrossRef]

8. Karacam Z, Eroglu K. Effects of Episiotomy on Bonding and Mother's Health. J Adv Nur 2003; 43: 384-94.

9. Şahin NH, Yıldırım G, Aslan E. Evaluating the Second Stages of Deliveries Maternity Hospital. Turkiye Klinikleri J Gynecol Obstet 2007; 17: 37-43.

10. Sayıner FD, Demirci N. Prenatal Perineal Masajın Vaginal Doğumlarda Etkinliği, iÜFN Hem Derg 2007; 15:146-54.

11. ACOG. Ob-Gyns Can Prevent and Manage Obstetric Lacerations During Vaginal Delivery, Says New ACOG Practice Bulletin.2016 http:// www.acog.org/About-ACOG/News-Room/News-Releases/2016/ Ob-Gyns-Can-Prevent-and-Manage-Obstetric-Lacerations

12. RCOG. Episiotomy. 2012 https://www.rcog.org.uk/en/ guidelines-research-services/guidelines/episiotomy---query-bank/

13. SOGC. Guidelines For Operative Vaginal Birth. 2004; No: 148. https:// sogc.org/wp-content/uploads/2013/01/148E-CPG-August2004.pdf

14. Berkowitz LR, Foust-Wright CE. Approach to Episiotomy, UpToDate 2016 https://www.uptodate.com/contents/ approach-to-episiotomy/print

15. Carroli G, Mignini L. Episiotomy for Vaginal Birth. Cochrane Database Syst Rev 2009; 21: CD000081. [CrossRef]

16. Joshi A, Acharya R. Perineal Outcome After Restrictive Use of Episiotomy in Primigravidas. J Nepal Med Assoc 2009; 48: 269-72.

17. Albers LL, Sedler KD, Bedrick EJ, Teaf D, Peralta P. Midwifery Care Measures in the Second Stage of Labor and Reduction of Genital Tract Trauma at Birth: A Randomized Trial. J Midwifery Womens Health 2005; 50: 365-72. [CrossRef]

18. Mei-dan E, Walfisch A, Raz I, Levy A, Hallak M. Perineal Massage During Pregnancy: a Prospective Controlled Trial. Isr Med Assoc J 2008; 10: 499-502.

19. Attarha M, Vakillian K, Rozbahany N, Bekhradi R. Effect of Perineal Massage with Lavender Essence on Episiotomy and Laceration. J Babol Univ Med Sci 2009; 11: 25-30.

20. Aasheim V, Nilsen AB, Lukasse M, Reinar LM. Perineal Techniques During the Second Stage of Labour for Reducing Perineal Trauma. Cochrane Database Syst Rev. 2011; 7: CD006672. [CrossRef]

21. Foroughipour A, Firuzeh F, Ghahiri A, Norbakhsh V, Heidari T. The Effect of Perineal Control with Hands-on and Hand-poised Methods on Perineal Trauma and Delivery Outcome. J Res Med Sci 2011;16:1040-6.

22. Fahami F, Shokoohi Z, Kianpour M. The effects of Perineal Management Techniques on Labor Complications. Iran J Nurs Midwifery Res 2012; 17: 52-7.
23. Beckmann MM, Stock OM. Antenatal Perineal Massage For Reducing Perineal Trauma. Cochrane Database Syst Rev 2013; 4: CD005123. [CrossRef]

24. Rozita R, Saatsaz S, Chan YH, Nia HS. A Comparison of the "HandsOff" and "Hands-On" Methods to Reduce Perineal Lacerations: A Randomised Clinical Trial. J Obstet Gynaecol India 2014; 64: 425-9. [CrossRef]

25. Zare O, Pasha H, Faramarzi M. Effect of Perineal Massage on the Incidence of episiotomy and Perineal Laceration. Health 2014;6:104. [CrossRef]

26. Demirel G, Gölbaşı Z. Effect of Perineal Massage on the Rate of Episiotomy and Perineal Tearing. Int J Gynaecol Obstet 2015;131:183-6. [CrossRef]

27. Lemos A, Amorim MM, Dornelas de Andrade A, de Souza Al, Cabral Filho JE, Correia JB. Pushing/bearing down Methods for the Second Stage of Labour. Cochrane Database Syst Rev 2015; CD009124. [CrossRef]

28. Lodge F, Haith-Cooper M. The Effect of Maternal Position at Birth on Perineal Trauma: A Systematic Review, BJM 2016; 24: 172-80. [CrossRef]

29. Warmink-Perdijk WDB, Koelewijn JM, de Jonge A, van Diem MT, LagroJanssen ALM. Better Perineal Outcomes in Sitting Birthing Position cannot be Explained by Changing from Upright to Supine Position for Performing an Episiotomy. Midwifery 2016; 34: 1-6. [CrossRef]

30. Prins M, Boxem J, Lucas C, Hutton E. Effect of Spontaneous Pushing versus Valsalva Pushing in the Second Stage of Labour on Mother and Fetus: A Systematic Review of Randomised Trials. BJOG 2011;118: 662-70. [CrossRef]

31. Diorgu FC, Steen MP, Keeling JJ, Mason-Whitehead E. Mothers and midwives perceptions of birthing position and perineal trauma: An exploratory study. Women Birth 2016; 9: 518-23. [CrossRef]

32. Taşkın L. Riskli Doğum Eylemi. İçinde Doğum ve Kadın Sağlığı Hemşireliği. Taşkın L. Edt. 11. Baskı. Ankara: Sistem Ofset Mabaacılık, 2012: ss:370-1.

33. Ballesteros-Meseguer C, Carrillo-García C, Meseguer-de-Pedro M, Canteras-Jordana M, Martínez-Roche ME. Episiotomy and its Relationship to Various Clinical Variables that Influence its Performance. Rev Lat Am Enfermagem 2016; 24: e2793. [CrossRef]

34. Kwon HY, Park HS. Episiotomy and the Risk of Severe Perineal Injuries Among Korean Women. J Matern Fetal Neonatal Med 2017;30:174549. [CrossRef]

35. Dahlen HG, Homer CS, Cooke M, Upton AM, Nunn R, Brodrick B. Perineal Outcomes and Maternal Comfort Related to the Application of Perineal Warm Packs in the Second Stage of Labor: A Randomized Controlled Trial. Birth 2007; 34: 282-90. [CrossRef]

36. Gupta JK, Nikodem VC. Woman's Position During Second Stage of Labour. Cochrane Database Syst Rev 2000; 2: CD002006. [CrossRef]

37. Kalis V, Stepán JJr, Králícková M, Zlůvová P, Rokyta Z. Maternal Position at the Delivery and Perineal Trauma. Ceska Gynekol 2007;72:241-6.

38. Gupta JK, Hofmeyr GJ, Shehmar M. Position in the Second Stage of Labour for Women Without Epidural Anaesthesia. Cochrane Database Syst Rev 2012; 5: CD002006. [CrossRef]

39. Meyvis I, Rompaey BV, Goormans K, Truijen S, Lambers S, Mestdagh E et al. Maternal Position and Other Variables: Effects on Perineal Outcomes in 557 Births. Birth 2012; 39: 115-20. [CrossRef]

40. De Souza Caroci da Costa A, Gonzalez Riesco ML. A Comparison of "Hands off" versus "Hands on" Techniques for Decreasing Perineal Lacerations During Birth. J Midwifery Womens Health 2006;51:10611. [CrossRef]

41. Berghella V. Obstetric Evidence Based Guidelines. London: Informa Healthcare, 2007: 60.

42. Varney H, Kriebs JM, Gegor CL. Varney's Midwifery 4th edition. London: Jones and Bartlett Publishers, 2004: 842-3. 